\title{
Analysis of cytotoxic activity of peripheral blood natural killer cells in women with recurrent miscarriage
}

\begin{abstract}
Around $50 \%$ of recurrent spontaneous abortions (RSA) remain unexplained. Immunological etiology has been proposed, supported by evidence of lower count of natural killer (NK) cells in peripheral blood of RSA women compared to women with normal delivery history. However, studies concerning the cytotoxic activity of NK cells in women with RSA are still controversial. We performed an observational casecontrol study assaying peripheral blood NK ( $\mathrm{pNK}$ ) cells cytotoxic activity in nonpregnant RSA women compared to non-pregnant women with normal delivery history. Twelve RSA and nine control women were recruited and blood samples were drawn during the luteal phase of ovarian cycle. pNK cells were incubated with target CFSElabeled K562 cells and cytotoxicity was measured by cytofluorimetry. In non-pregnant RSA women pNK cytotoxic activity was not significantly altered compared to control women.In luteal phase of ovarian cycle the level of cytotoxic activity of pNK cells is not a marker for predicting RSA, and clinicians should not use pNK activity as a systematic recurrent pregnancy loss examination.
\end{abstract}

Keywords: spontaneous abortion, recurrent miscarriage, natural killer cells, cytotoxicity, cytofluorimetry
Volume 4 Issue 3 - 2018

\author{
Roberta Capucci,' Paola Franceschelli, ${ }^{2}$ \\ Simona Carboni,' Alfredo Patella,' Rosaria \\ Cappadona,' Rita Bassi Andreasi, ${ }^{2}$ Michele \\ Rubini $^{2}$ \\ 'Department of Reproduction and Development, Sant'Anna \\ University Hospital, Italy \\ ${ }^{2}$ Depatment of Biomedical and Specialty Surgical Sciences, \\ University of Ferrara, Italy
}

\begin{abstract}
Correspondence: Michele Rubini, Department of Biomedical and Specialty Surgical Sciences, University of Ferrara,Via Fossato di Mortara 74, 44I2 I Ferrara, Italy, Tel +39-0532-9744-73, Fax +39-0532-236157, Email rub@unife.it
\end{abstract}

Received: January 02, 2018 | Published: June 12, 2018
Abbreviations: RSA, recurrent spontaneous abortions; pNK, peripheral blood natural killer cells; uNK, uterine natural killer cells; BMI, body mass index

\section{Introduction}

About $1 \%$ of couples who are trying to conceive will suffer recurrent spontaneous abortion (RSA), defined as three or more subsequent pregnancy loss before 20 weeks of gestation. ${ }^{1,2}$ Several causes have been proposed for RSA, including chromosomal abnormalities, antiphospholipid syndrome, uterine malformations, endocrine defects, and cervical incompetence.

Although at least one of these causes is present in about half of patients, in the other half RSA remains completely unexplained. ${ }^{3} \mathrm{~A}$ number of evidence suggests that innate immunity could play a role in the etiology of unexplained RSA. ${ }^{4}$ In particular, natural killer (NK) cells, a major component of the innate immunity, are the predominant immune cells present at the maternal-fetal interface during early pregnancy and some evidence suggest that the activity of these uterine NK cells ( $\mathrm{uNK}$ ) in decidual tissue could influence pregnancy success. ${ }^{5-7}$

NK cells play a central role in the early defenses against viral infection acting through cytolytic activity secretion of proinflammatory cytokines. ${ }^{8}$ Around $90 \%$ of peripheral blood NK (pNK) cells are CD16+ CD56dim and have high cytotoxic activity, while the CD16- CD56 bright subgroup is the main source of NK cellderived immuno-regulatory cytokines. ${ }^{9}$ uNK cells derive from this latter subpopulation, promote placental and trophoblast growth, and provide immunomodulation at the maternal-fetal interface. ${ }^{6}$ Evidence suggests that NK cells number and cytotoxicity level are hormonally regulated. During the luteal phase of the ovarian cycle uNK number, pNK cells number and cytotoxicity are increased compared to the follicular phase. ${ }^{10,11}$ In normal human early pregnancy, progesterone causes decreasing in pNK cell number and activity, and recruitment of uNK cell by cytokines secreted from endometrial stromal cells, which also regulate uNK cell proliferation and differentiation, ultimately supporting local immunomodulation and placenta development. ${ }^{6,12}$ Dysregulation of NK cells has been hypothesized to play a role in RSA, as initially suggested from evidence of increased number of pNK cells in women with RSA history compared to control women ${ }^{13,14}$ and preconceptional NK activity was suggested as predictor of miscarriage in Japanese patients with RSA. ${ }^{15}$ However, studies on the association between pNK cytotoxicity and RSA show controversial results, ${ }^{16-18}$ and more recently a large cohort study in Japan reported no association between pNK cytotoxic activity and risk of subsequent miscarriage in women with RSA. ${ }^{19}$

pNK cell number and pNK cell cytotoxicity do not strictly correlate, as it is possible to have significant pNK activity even in presence of low pNK cell count. As regards to the uterine compartment, it was hypothesized that the activity level of uNK cells present in the lining of womb during early pregnancy could influence the embryo survival, rather than their absolute cell count. It is not clear, however, if this applies also to pNK cells, i.e. if activity level of pNK cell associates with risk of spontaneous abortion. ${ }^{20}$

The aim of the present observational case-control study is to investigate if the level of pNK cytotoxic activity associates with RSA in Italian population, comparing a group of RSA women with a group of matched control women with normal pregnancy history.

\section{Materials and methods}

Study subjects were recruited at the Gynecology and Obstetrics Units of the S. Anna University Hospital of Ferrara, Italy, during year 2014. A total of 12 women with history of at least two 
idiopathic spontaneous miscarriage with the same partner and a desire for pregnancy were selected. A miscarriage was defined as a spontaneous pregnancy loss before 22 weeks of gestation. Women with recurrent abortions presumably related to specific pathologies, such as immunological diseases, uterine malformation or hormonal dysfunction, were excluded from the study. Age, body mass index (BMI) and obstetrical history of the women included in the study are reported in Table 1. A control group of 9 women with a history of normal pregnancy and delivery was recruited in the same period. This study was approved by Local Ethical Committee (CE-BIF 24.10.2013), and all subjects included in the study provided a written informed consent before their participation, in agreement with the World Medical Association Declaration of Helsinki.

Table I Age, BMl; and obstetrical history of women with idiopathic spontaneous abortion included in the study

\begin{tabular}{lll}
\hline & Mean \pm SD $\dagger$ & Range \\
\hline Age $\ddagger$ & $35.5 \pm 4.3$ & $29-45$ \\
BMI $\S$ & $23.8 \pm 5.6$ & $18.5-34.0$ \\
N $^{\circ}$ SA $\uparrow$ & $3.3 \pm 3.0$ & $2-6$ \\
Parity & $0.2 \pm 0.4$ & $0-1$ \\
\hline
\end{tabular}

$\dagger$, standard deviation; $\ddagger$, age in years; §, BMI: body mass index; $\llbracket S A$, number spontaneous abortion events

From all participating subjects a peripheral blood sample was drawn during the luteal phase of ovarian cycle, as assessed by a gynecology specialist. Peripheral blood mononuclear cells (PBMCs) were isolated by density centrifugation. pNK cells cytotoxicity was estimated after co-culturing PBMC with carboxyfluorescein succinidimyl ester (CSFE)-labelled K5632 target cells, and detecting cell death by propidium iodide (PI) staining using flow-cytometry. ${ }^{21}$ K562 cells are from erythromyelocytic leukemia, are specifically killed by NK cells but not by other cells. K 562 cells were maintained in RPMI 1640 with L-glutamine and $\mathrm{NaHCO}_{3}$ (Sigma-Aldrich, St. Louis, USA), supplemented with 10\% Fetal Bovine Serum (Gibco/ Invitrogen, Karlsruhe, Germany), 100IU/ml penicillin and $100 \mu 1 / \mathrm{ml}$ streptomycin (Gibco/Invitrogen, Karlsruhe, Germany) at 5\% $\mathrm{CO}_{2}$, $37^{\circ} \mathrm{C}$. One day prior to cytotoxic assay cells were sub-passaged to ensure growth in the log phase. After two washing with cold phosphate buffered solution (PBS), K562 cells were resuspended at $5 \times 10^{6} \mathrm{cells} / \mathrm{ml}$ concentration in pre-warmed PBS $\left(37^{\circ} \mathrm{C}\right)$, and labeled with $0.1 \mu \mathrm{M}$ CFSE (CellTraceTM CFSE Cell Proliferation kit, Molecular probes/Invitrogen, Eugene, Oregon) for $10 \mathrm{~min}$ at room temperature in the dark. CFSE penetrates plasma membranes and covalently binds cytoplasmic proteins, yielding green fluorescent target cells. The reaction was stopped by adding 4-5 volumes of RMPI 1640 containing $10 \%$ FBS and incubating on ice for 5 minutes. CFSElabeled K562 cells were washed three times with RMPI 1640 with $10 \%$ FBS and resuspended at the final concentration of $1 \times 10^{6}$ cells/ $\mathrm{ml}$. Cell viability, as assessed by Trypan Blue dye exclusion assay, was always over $95 \%$.

Effector cells (PBMCs) were isolated from $5 \mathrm{ml}$ of heparinized peripheral blood samples by density gradient centrifugation on Lympholyte (Cederlane, Burlington, Ontario, Canada). PBMC were washed twice with RMPI 1640 and resuspended at $5 \times 10^{6}$ cells $/ \mathrm{ml}$ in RPMI 1640 containing 10\% FBS.

PBMCs and CFSE-labelled K562 cells were co-incubated in flat bottom 48-well sterile polystyrene plates (Costar corporation,
Cambridge, Massachusetts, USA) at an effector/target (E:T) ratio of $10: 1$. Cultures were incubated at $37^{\circ} \mathrm{C}$ in $5 \% \mathrm{CO} 2$ atmosphere for 4 hours. Target cells were assayed separately to determine the background level of spontaneous cell death. As positive control reference, the maximum NK activity was obtained by stimulating effector cells with $2.5 \mu \mathrm{g} / \mu 1$ phorbol 12-myristate 13-acetate (PMA, Sigma-Aldrich, St. Louis, USA) and $0.5 \mu \mathrm{g} / \mu 1$ Ionomycin (SigmaAldrich, St. Louis, USA) one hour prior to adding target cells.

After co-incubation, cells were treated with PI (Sigma-Aldrich, St. Louis, USA) at final concentration of $1 \mu \mathrm{g} / \mathrm{ml}$ to label dead target cells. After 15 min of incubation on ice in dark, samples were analyzed using BD FACSCalibur flow cytometer and CellQuest Software (BD Biosciences, Franklin Lakes, NJ, USA). The percentage of cytolytic activity was determined by gating the target cells population in a FL1 dot plot (CFSE green fluorescence) versus FL3 (PI red fluorescence). Data for at least 2000 target cells were analyzed for each test.

The cell-mediated cytolytic activity of pNK cells was expressed as percentage of dead target cells. Each experiment was done in duplicate, and background values subtracted. Results were expressed as average values and $95 \%$ confidence interval after normalization to PMA/Ionomycin reference. Comparison of results obtained in RSA and control women was done by "two sample t-test between percents" using Statistic Calculator software version 4.0 (StatPac Inc., Pepin, WI, USA).

\section{Results and discussion}

In assays carried using PBMC from RSA or control women the stimulation with PMA/Ionomycin induced cytolysis of 55.6\%. This value can be taken as the maximal pNK cytolytic activity of the assay. Cytotoxic activity of NK from PBMCs from RAS patients or controls using K562 as target is reported in Figure 1A. Among the 12 RSA patients included in the study mean value of pNK cell cytotoxic activity was $3.73 \%$ (95\% CI 2.94-4.52) (Figure 1B), while resulted on average of $4.30 \%$ (95\% CI 3.44-5.17) among the 9 control women (Figure 1C). The two mean values resulted not significantly different, as case-control comparison using "two sample t-test between percents" turned out with t-statistic of 0.067 and a P-value of 0.947 . Moreover, no association was observed between age and pNK cells cytotoxic activity among RSA patients, nor among control women (data not shown).

NK cells are the most abundant population of lymphocyte present at maternal-fetal level and play a fundamental role in implantation process and in pregnancy outcome. ${ }^{13}$ Increased activity of NK cells has been suspected to be involved in rejection of the fetus by preventing trophoblast invasion of the endometrium. ${ }^{22}$ Several studies investigated alteration of NK cells cytotoxic activity or cell number as cause of RSA or other pregnancy-related pathological conditions, but results are still controversial. Some investigations, focusing on uNK, report no difference in total uNK number in women with RSA compared with women with normal delivery history, ${ }^{23,24}$ although a trend for a decrease number of CD16-CD56 bright NK cell subset compared to the number of CD16+CD56dim NK cells was observed. ${ }^{23}$ This effect could be related to an increase in NK number or activity in peripheral blood, causing a higher recruitment of CD16-CD56bright NK cell in the endometrium. ${ }^{6}$

In the present study, based on of PI staining of CSFE-labelled K562 target cells and cytofluorimetric detection, no significant difference in 
pNK cells cytotoxic activity was found in RSA compared to control women. This result is in agreement with Souza et al., ${ }^{16}$ that used a chromium-51 release cytotoxicity assay and reported no difference in pNK cell cytotoxicity between a group of 9 RSA patient and a control group of 9 women, and with a Dutch study based on 43 RSA patients and 37 controls. ${ }^{24}$ Our results also replicate well the findings of Nagoya's study that used the same chromium-51 release cytotoxicity assay and included a large cohort of 552 RSA cases. ${ }^{19}$ However, a study carried out in Iran on 23 RSA cases and 36 controls but based on lactate dehydrogenase $(\mathrm{LDH})$ release assay resulted with significantly higher pNK cytotoxic activity among RSA women compared to controls, along with an increased number of CD56dim cells. ${ }^{18}$ In this latter study the secretory phase of ovarian cycle of women included in the study was defined by self-reporting instead of determined by a gynecologist, and this could have influenced the study outcomes, but we cannot exclude that different results could be consequence of the diverse method used to assess pNK cytotoxic activity. Moreover, we cannot ignore that controversial results across studies could rely on differences in the environmental conditions to which different studied populations are exposed to, or to different genetic background.

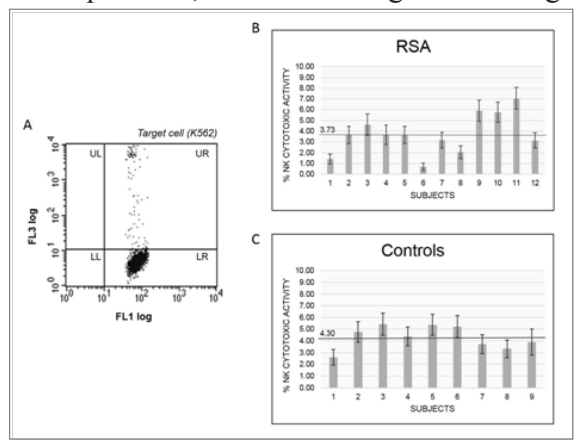

Figure I NK cytotoxic activity in RSA women compared to controls. (A) Flow-cytometry plot of CFSE-labelled target cells (K562) after incubation with PBMCs. NK cells activity is determined as the percentage of dead cells among total target cells by gating for the FLI (CFSE fluorescence) and FL3 (Propidium iodide fluorescence). UR: dead K562 cells; LR: live K562 cells. Relative cytotoxic activity is determined after normalization with maximal activity obtained by incubation of target cells with PMA/lonomycin. (B) Relative cytotoxic activity of NK cells isolated from peripheral blood samples of RSA women. (C) Relative cytotoxic activity of NK cells isolated from peripheral blood samples of control women.

\section{Conclusion}

We conclude that the cytotoxic activity of pNK cells assessed in Italian RSA cases and matched healthy controls by PI staining of CSFE-labelled K562 target cells and cytofluorimetric detection does not associate with RSA, and that this finding matches well the results obtained in other population using chromium-51 release cytotoxicity assay. According to our results, pNK cytotoxic activity level is not a marker for predicting RSA, and confirm that clinicians should not use pNK activity as a systematic recurrent pregnancy loss examination. ${ }^{19}$

Since pNK cytotoxic activity level does not predict uNK activity, that is generally less cytotoxic, and based on the evidence supporting a role of uNK cells in immune tolerance at level of decidua-trophoblast interface, we suggest that cytotoxic activity of uNK cells, rather than pNK cells should be studied in order to identify a predictive marker for RSA.

\section{Acknowledgements}

This study was partly supported by FAR-2016 grant from University of Ferrara.

\section{Conflict of interest}

The author declares that there are no conflicts of interest.

\section{References}

1. Stirrat GM. Recurrent miscarriage. Lancet. 1990;336:673-675.

2. Regan L. Recurrent early pregnancy failure. Curr Opin Obstet Gynecol. 1992;4:220-228.

3. Lee RM, Silver RM. Recurrent pregnancy loss: summary and clinical recommendations. Semin Reprod Med. 2000;18(4):433-440.

4. Van Wijk IJ, Van Vugt JM, Mulders MA, et al. Enrichment of fetal trophoblast cells from the maternal peripheral blood followed by detection of fetal deoxyribonucleic acid with a nested $\mathrm{X} / \mathrm{Y}$ polymerase chain reaction. Am J Obstet Gynecol. 1996;174(3):871-878.

5. Moffet-King A. Natural killer cells and pregnancy. Nat Rev Immunol. 2002;2:656-663.

6. Dosiou C, Giudice LC. Natural killer cells in pregnancy loss: endocrine and immunologic perspectives. Endocr Rev. 2005;26(1):44-62.

7. Vacca P, Mingari MC, Moretta L. Natural killer cells in human pregnancy. J Reprod Immunol. 2013;97(1):14-19.

8. Robertson MJ, Ritz J. Biology and clinical relevance of human natural killer cells. Blood. 1990;76(12):2421-2438.

9. Cooper MA, Fehniger TA, Caligiuri MA. The biology of human natural killer-cell subsets. Trends Immunol. 2001;22(11):633-640.

10. Bulmer JN, Morrison L, Longfellow M, et al. Granulated lymphocytes in human endometrium: histochemical and immunohistochemical studies. Hum Reprod. 1991;6(6):791-798.

11. Lee S, Kim J, Jang B, et al. Fluctuation of peripheral blood T, B, and NK cells during a menstrual cycle of normal healthy women. J Immunol. 2010;185(1):756-762.

12. Gregory CD, Lee H, Rees GB, et al. Natural killer cells in normal pregnancy: analysis using monoclonal antibodies and single-cell cytotoxicity assays. Clin Exp Immunol. 1985;62(1):121-127.

13. Kwak JY, Beaman KD, Gilman-Sachs A, et al. Up-regulated expression of $\mathrm{CD} 56+, \mathrm{CD} 56+/ \mathrm{CD} 16+$, and $\mathrm{CD} 19+$ cells in peripheral blood lymphocytes in pregnant women with recurrent pregnancy losses. $\mathrm{Am} J$ Reprod Immunol. 1995;34(2):93-99.

14. Thum MY, Bhaskaran S, Abdalla HI, et al. An increase in the absolute count of CD56dimCD16+CD69+ NK cells in the peripheral blood is associated with a poorer IVF treatment and pregnancy outcome. Hum Reprod. 2004;19(10):2395-2400.

15. Aoki K, Kajiura S, Matsumoto Y, et al. Preconceptional natural-killercell activity as a predictor of miscarriage. Lancet. 1995;345(8961):1340 1342 .

16. Souza SS, Ferriani RA, Santos CM, et al. Immunological evaluation of patients with recurrent abortion. J Reprod Immunol. 2002;56(12):111-121.

17. Emmer PM, Nelen WL, Steegers EA, et al. Peripheral natural killer cytotoxicity and CD56(pos)CD16(pos) cells increase during early pregnancy in women with a history of recurrent spontaneous abortion. Hum Reprod. 2000;15(5):1163-1169. 
18. Karami N, Boroujerdnia MG, Nikbakht R, et al. Enhancement of peripheral blood CD56(dim) cell and NK cell cytotoxicity in women with recurrent spontaneous abortion or in vitro fertilization failure. $J$ Reprod Immunol. 2012;95(1-2):87-92.

19. Katano K, Suzuki S, Ozaki Y, et al. Peripheral natural killer cell activity as a predictor of recurrent pregnancy loss: a large cohort study. Fertility and Sterility. 2013;100(6):1629-1634.

20. Gilman-Sachs A, DuChateau BK, Aslakson CJ, et al. Natural killer (NK) cell subsets and NK cell cytotoxicity in women with histories of recurrent spontaneous abortions. Am J Reprod Immunol. 1999;41(1):99-105.

21. Lecoeur H, Féfrier M, GarciaS, et al. A novel flow cytometric assay for quantification and multiparametric characterization of cell mediated cytotoxicity. J Immunol Methods. 2001;253(1-2):177-187.
22. Lunghi L, Ferretti ME, Medici S, et al. Control of human trophoblast function. Reprod Biol Endocrinol. 2007;5:6.

23. Lachapelle MH, Miron P, Hemmings R, et al. Endometrial T, B, and NK cells in patients with recurrent spontaneous abortion. Altered profile and pregnancy outcome. J Immunol. 1996;156(10):4027-4034.

24. Shimada S, Kato EH, Morikawa M, et al. No difference in natural killer or natural killer T-cell population, but aberrant T-helper cell population in the endometrium of women with repeated miscarriage. Hum Reprod. 2004;19(4):1018-1024. 